This item was submitted to Loughborough's Research Repository by the author.

Items in Figshare are protected by copyright, with all rights reserved, unless otherwise indicated.

\title{
Low-cost bus seating information technology system
}

PLEASE CITE THE PUBLISHED VERSION

https://doi.org/10.1049/iet-its.2019.0529

PUBLISHER

IET

VERSION

AM (Accepted Manuscript)

PUBLISHER STATEMENT

This paper is a postprint of a paper submitted to and accepted for publication in IET Intelligent Transport Systems and is subject to Institution of Engineering and Technology Copyright. The copy of record is available at the IET Digital Library.

\section{LICENCE}

CC BY-NC-ND 4.0

\section{REPOSITORY RECORD}

Murdan, Anshu Prakash, Vicky Bucktowar, Vishwamitra Oree, and Marcus Enoch. 2020. "Low-cost Bus Seating Information Technology System”. Loughborough University. https://hdl.handle.net/2134/13103579.v1. 


\title{
A low-cost Bus Seating Information Technology (BusSIT) system
}

\author{
Anshu Prakash Murdan ${ }^{1 *}$, Vicky Bucktowar ${ }^{2}$, Vishwamitra Oree ${ }^{3}$, Marcus P Enoch ${ }^{4}$ \\ 1,2,3 Department of Electrical and Electronic Engineering, University of Mauritius, Reduit, 80837, Republic of \\ Mauritius \\ ${ }^{4}$ School of Architecture, Building and Civil Engineering, Loughborough University, Leicestershire LE11 3TU, \\ UK \\ *a.murdan@uom.ac.mu
}

\begin{abstract}
Public transport operators often struggle to provide a reliable and efficient transport service. A lack of comprehensive real-time operational data is often cited as a major cause for this state of things. In this paper, we report on the design, implementation and testing of an Internet of Things-based system, named Bus Seating Information Technology (BusSIT) system, which dynamically determines vehicle occupancy while the bus is in service. It uses an array of sensors for detecting events in the vehicle: infrared sensors ascertain whether passengers are entering and leaving the bus; force sensitive resistors facilitate seatoccupancy detection; a Global Positioning System shield in conjunction with a Raspberry Pi 4 microcomputer enable real-time tracking of the bus; and a USB camera connected to the same Raspberry Pi assist in cross-checking and validating the preceding information. The data collected is uploaded to an online IoT platform (thinger.io), through $3 \mathrm{G}$ or $4 \mathrm{G}$ if available, and can be visualized via an android app as well as through a desktop computer user interface. The planned functions of the system were tested in a 20 -seater bus. Results showed that the system is able to track the vehicle location, as well as vehicle occupancy in realtime in most cases.
\end{abstract}

Keywords: passenger count; vehicle occupancy; real-time; microcontroller; vehicle tracking

\section{Introduction}

Understanding how many people are using a public transport service at any particular time and at specific points on a network is of vital importance for passengers and service providers alike. Public transport congestion can result in long waiting times, denied boardings and lower service reliability [1]. Consequently, alleviating congestion has been one of the main objectives of public transport authorities and stakeholders. According to Hensher et al. [2], crowding curtailment is a leading consideration in the design of new stations for public transport systems. Moreover, several studies have empirically estimated that public transport passengers are willing to pay more for crowding reduction [3]. In this context, timely and reliable information on seat occupancy in public transport services could lead to passengers taking better informed decisions about how, when, where and/or whether they travel. From an operator perspective, the short-term benefits of such information include more rapid responses to unexpected events. In the longer term, seating occupancy data helps operators to better identify changing travel markets so that they can adapt their service provision accordingly. Finally, government authorities benefit from a better understanding of the travel preferences of their citizens, enabling them to improve the effectiveness of subsidies, investments and other policy decisions.

Yet in practice, knowledge of passenger loads by public transport operators is often far from comprehensive [4]. A survey carried out among 86 transit agencies in the United States (US) revealed that 24 of them relied exclusively on automated passenger counters (APCs) and a further 44 used a mixture of automated and manual systems [5]. Among those equipped with APCs, only a fifth generated daily ridership reports while merely a tenth looked at stop-level boardings on a daily basis. In addition, the data is rarely passed on to passengers after it is collected. For example, another study of 29 transit agencies in the US showed that only three of them routinely provided information on seat availability or crowdedness to passengers [6]. The most commonly cited justification for not installing APCs is their high cost, with an average capital cost of USD 6,638 and an additional yearly amount of USD 600 for operation and maintenance purposes [5]. Furthermore, automatic collection of passenger occupancy data is not a priority for most service providers [5].

Consequently, public transport services are often planned on the basis of information obtained at the route or network level rather than at the stop level, resulting in data that is insufficiently disaggregated. Besides, the data is incomplete as it is acquired at discrete time intervals rather than continuously. Finally, the data does not reflect the realtime situation as it is usually derived from historical studies. In the past, these shortcomings coupled with the high cost associated with the collection and analysis of high volumes of good quality data made such management decisions understandable. However, the falling costs and rapid developments in sensor and information and communication technologies imply that such decisions are less reasonable today.

\section{Aim}

In light of the above, this paper proposes the design and implementation of an instrumented bus to remotely determine the number of people travelling in near real-time whilst also capturing useful data about passenger origins and destinations. The proposed low-cost vehicle occupancy system is based on inexpensive microcontrollers and sensors along with an open 
source online IoT platform. The main design criteria of the system pertain to its affordability, reliability and userfriendliness. In addition, the real-time data captured by the system can be automatically archived in an appropriate format to facilitate future analysis.

\section{Literature Review}

Several approaches have been adopted by the public transport industry to determine the number of passengers on buses. Over the years, technologies have evolved from basic manual counting methods to automated approaches that involve the use of handheld units, electronic registering fareboxes (ERFs) and APCs. Electronic Ticketing Machines (ETMs) are gaining popularity as they can be used to acquire temporal or spatial variation data in ridership as well as information about the type of passenger (student, pass user, senior citizen, etc.). Similarly, Global Positioning Systems (GPS), smart cards and transponders constitute other common means to obtain information about transit and passenger characteristics. In view of the growing significance of route and sub-route passenger numbers in identifying opportunities to reallocate scarce resources in an increasingly constrained economic environment, present-day public transport operators have a wide array of methods at their disposal to count their passengers. Existing vehicle occupancy measuring approaches vary in complexity, strategy, cost and accuracy.

In recent years, novel machine learning techniques as well as latest technologies like contactless bank cards and Near Field Communication (NFC) have been employed in transit systems [7], for fare payment and/or passenger count. More recently, deep learning has been used for a tour bus seat selection. The authors used neural network architectures like ResNet-152, DenseNet-161, and Inception v3. The proposed system named Beautiful Scenery \& Cool Shade provided tour bus passengers information about scenery and sunlight in four directions using deep learning and the azimuth theory [8]. Regarding black carbon (BC) concentrations on bus seats, Bauer et al. [9] found that there was no difference in the mean $\mathrm{BC}$ concentrations in different seating areas, however the frequency of BC peaks was significantly higher in some priority areas. It was also deduced that open doors near green spaces or away from motorized traffic reduce BC concentration.

\subsection{Passenger counting systems}

Several techniques have been applied to measure vehicle occupancy by counting the passengers at various points while they make their way to the vehicle. They include automated ticket barriers or entry-exit barrier-based systems such as ETMs or manual clickers to count people at a ticket line and treadle mats. Ticketing data has been found to be a major means of recording public transport passenger numbers around the world [10]. Wei et al. [11] demonstrated the potential of publicly available data derived from ticketingwebsite for the Chinese high-speed rail, in order to retrieve information on unused transit capacity, occupancy rate of trains, and passenger flux at stations. The most common automated entry-exit counting technologies are infrared (IR) beams, whereby passengers interrupt two beams in a particular sequence. In doing so, they activate an APC device into registering a boarding or alighting traveller. ERFs also contribute to a large extent to the pool of ticketing data by making use of technologies like magnetic strip cards, contacttype integrated circuit smart cards and proximity cards. Smart cards, in particular, have been widely adopted worldwide during the last decade. Although their primary function is to collect revenue, they also generate a huge amount of data about the operation of transit systems. Besides counting passengers, the spatio-temporal information contained in this continuous flux of data can be analysed to reveal a wide range of aspects relating to passenger behaviour. In this context, several studies have used smart card data to analyse passenger travel patterns in view of improving the planning and operation of the transport network [12], [13]. Smart card data mining has also been found to be valuable in estimating the travel demand at various times of the day and accordingly adjust the scheduling of vehicles [14]. Nevertheless, validation of the findings of the studies that use smart card data is difficult. Since the data is always anonymous, it is extremely challenging to interpret and understand any transit patterns observed [15].

Treadle mats, on the other hand, are triggered when they are stepped upon by passengers. Baltes and Rey [16] presented the results of a survey of North American transit systems related to APC usage, and summarized critical issues for transit systems to consider. In particular, the authors highlighted the importance of having a detailed route inventory and the ability to analyse large amount of data for precise analysis of passenger activity. Rakebrandt [17] noted that the success of manual passenger counting programs by several transit agencies in the US demonstrate the much greater importance of establishing appropriate procedures instead of only focusing on the choice of technology used.

\subsection{Indirect Detection Systems}

Besides passenger counting methods, other schemes are used to determine the number of people in vehicles. They range from systems that detect changes in vehicle weight or thermal intensity and passive data streams to those based on closed-circuit television (CCTV) image processing. One example of such systems is the Siemens Class 700 Desiro trains, used by Greater Thameslink Railway Services in the UK [18]. The passenger occupancy is estimated using a 'load weigh' system, whereby scales are fitted to the wheel bogies and the results are communicated to the users via the Passenger Information System. Hashimoto et al. [19] developed a thermal system which counted the number of people, and also detected their directions of movement near a $200 \mathrm{~cm}$ wide door, achieving an accuracy of $95 \%$ accuracy. Trépanier and Yamamoto [20] reviewed various methodologies used to process voluminous passive data streams collected from technologies such as mobile phones, GPS devices and Bluetooth sensors, in order to analyze the behaviour of transport users. However, while highlighting their huge potential, the authors also pointed out the major issues associated with these approaches, particularly data privacy and external model validation. Along the same lines, Oransirikul et al. [21] demonstrated the feasibility of measuring passenger loads using Wi-Fi signals from mobile devices. 


\subsection{Seat Occupancy Systems}

A more direct way to measure vehicle occupancy is to detect whether the seats are occupied or not, using different types of sensors. Zeeman et al. [22] described a low-cost capacitive proximity sensor which is fitted to each seat to determine its occupancy. Signals from all sensors are input to a microcontroller for onward transmission to a gateway unit via Zigbee module and is subsequently stored on a database. Nguyen et al. [23] combined a capacitance sensor with an infrared sensor (IR) to propose a low-cost and effective solution that not only detects seat occupancy but also solves the problem of seat hogging, in the context of a campus library. The IR sensor detects occupancy while the capacitive sensor establishes whether the occupant is human. However, the seat hogging solution is an indirect one as the capacitive sensor is placed on an adjacent table rather than on the chair. As such, the system is applicable to places like libraries and restaurants but cannot be used in buses. On the other hand, Atayero et al. [24] proposed 'SeatSense' based on a flexible force sensing resistor. The resistance of the latter varies with seat occupancy, dropping to less than $20 \Omega$ when the seat is occupied and in excess of $200 \mathrm{M} \Omega$ when the seat is vacant. A different methodology based on antenna detection was adopted by Sterner et al. [25]. The underlying principle relies on the attenuation of high frequency radio waves caused by the presence of a person between a pair of transmitting and receiving antennas integrated in a seat. The authors claimed that their proposed sensors are more accurate, easier to install and operate than pressure or force sensors.

\subsection{Camera systems for passenger counting}

Camera systems have also been used to count the number of passengers in vehicles. Thus, Chen et al. [26] proposed an automatic people counting system based on processing of images captured at entry and exit points of buses. Experimental results showed that the proposed buspassenger counting algorithm provided a count accuracy of $92 \%$ on average. Another mechanism for counting and monitoring passengers, both adults and children, at the entrance of buses was implemented using a RGB-D sensor installed over the bus door [27]. The accuracy and effectiveness of the proposed method were confirmed by experiments conducted in a real scenario. This approach has the advantage of being computationally inexpensive. In addition, the algorithm is flexible enough to obtain statistical measures on the number of people present in the bus in realtime. For this purpose, an Analytical Processing System accesses the database to extract statistical information about the bus passengers. Yang et al. [28] improved the passengercounting algorithm by enabling it to count passengers in a highly crowded situation at the entrance of a traffic bus. The unique characteristics of this single camera vision system included a novel feature-point tracking and an online clustering-based passenger counting framework. It also used a simple and highly accurate clustering algorithm that projected the high-dimensional feature-point trajectories into a 2-D feature space by their appearance and disappearance times. This enabled the counting of the number of people through online clustering. The results showed that the system could process two $320 \times 240$ video sequences at a frame rate of $25 \mathrm{fps}$ simultaneously, and could count passengers reliably in various difficult scenarios with complex interaction and occlusion among people. The method achieved high accuracy rates up to $96.5 \%$.

\subsection{Vehicle tracking technologies}

Often, vehicle occupancy data is more informative if it is coupled with vehicle location data. It is therefore interesting to also review experiences of vehicle tracking technologies. The possibility of monitoring a vehicle through travelled routes using a web-based system has been demonstrated in several works. For example, a vehicle tracking system using GPS and GSM technologies within a web application was displayed accurately on Google Map [29]. Elmedany et al. [30] presented another low-cost, realtime tracking system that provided accurate localizations of vehicles. The system was implemented using a GM862 cellular quad-band module. A monitoring server and a graphical user interface on a website were also implemented using Microsoft SQL Server 2003 and ASP.net to view the current location of a vehicle on a specific map. The security aspect of transportation was also highlighted in a system that enabled vehicles throughout a city to be monitored for accidents and robberies [31]. This was accomplished by using GPS for vehicle location and GSM for transferring data online. Along the same lines, Lita et al. [32] proposed a lowcost automotive localization system using GPS and GSMSMS services, which displayed the location of the vehicle on the driver's mobile phone as a SMS, upon his request. The designed system was also interconnected with the car alarm system in order to alert the owner in case of car theft.

Most of these works make use of GPS devices to track vehicles in real-time. Besides, the GPS service provider usually offers a platform to do standard analyses. Thus, it can generate common reports that summarize crucial information regarding the trips, including daily over-speeding reports and daily on-time performance reports. However, the main benefit of GPS data lies in the possibility of using the raw data generated by the system to perform pertinent analyses that can provide an improved understanding of key operational characteristics of the vehicle. The main data outputs from a GPS device are spatial positioning and time at location.

\subsection{Research gap}

It can be inferred from the foregoing literature review that existing research primarily focuses on passenger counting and vehicle tracking systems as two independent problems. There is a lack of scientific studies for a more comprehensive and integrated solution that encompasses passenger count, passenger seating preferences, as well as vehicle tracking. This research work attempts to bridge this gap by presenting a low-cost, integrated public transportation information system, named Bus Seating Information Technology (BusSIT). Its main features include passenger counting, seat occupancy detection, vehicle tracking and passenger behaviour monitoring.

\section{Methodology}

The BusSIT system consists of four major sub-systems, namely, an IR Passenger Counting System, a Seat Occupancy Detection System, a Bus Tracking System (via a GPS tracker), and a Real-Time Video Monitoring System (via a camera). Figure 1 shows the interactions among the main elements of the whole system. 


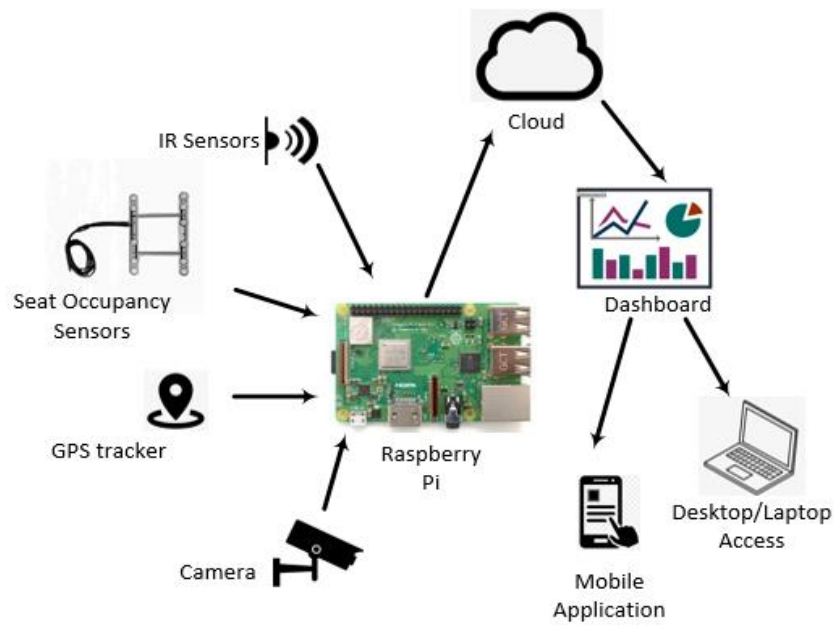

Fig. 1. Block diagram of BusSIT system

One advantage of this integrated system is that each block is designed to work independently irrespective of the status of the other blocks. However, if the Raspberry Pi, which is the heart of the system, becomes somehow faulty, the whole system collapses, since it is there will be no processing, nor any transmission to the cloud.

Each sub-system connects to cloud services and upload its data in real-time to the cloud. The raw data is processed and presented in a dashboard that can subsequently be viewed through either a desktop application or an Android smartphone application.

\subsection{Solution Architecture}

Figure 2 shows the overall architecture of the BusSIT system.

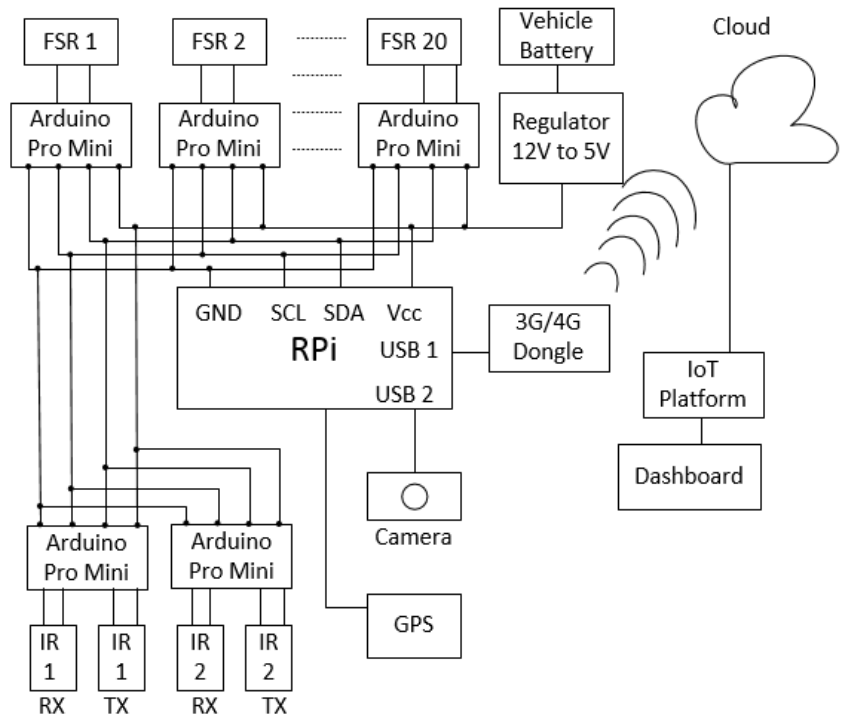

Fig. 2. Solution Architecture of the BusSIT system

The four main blocks, namely, the Seat Occupancy System, the IR Passenger Counting System, the Geolocation System and the Real Time Video Surveillance System are all connected to the Raspberry $\mathrm{Pi}$ microcomputer, through different ports. The latter is connected to the cloud through a
3G/4G network by means of a USB dongle. The following sections provide a description of the sensors, the software platforms, the different functional blocks and their integration with the Raspberry Pi. Open source hardware, software and IoT platforms have been favoured as far as possible in order to keep the proposed BusSIT system affordable.

\subsection{Hardware and Software Platforms}

\section{Raspberry Pi 4}

The Raspberry Pi is a small single-board computer that was released in 2012. Since then, its powerful processing capability and small size coupled with its affordability, expansion options and low power consumption have made it widely popular among developers. A recent version, the Raspberry Pi 4 Model B, was selected to process data locally and to control tasks within the bus. It features a Broadcom system on a chip (SoC) that includes a Quadcore ARM Cortex-A72 64-bit central processing unit (CPU) and an onchip graphics processing unit [33]. Furthermore, the on-board Wi-Fi and Bluetooth support as well as 4 GB RAM make the Raspberry Pi 4 Model B well suited for the proposed BusSIT system. It also offers a wide range of connectivity options with four USB slots, HDMI and composite video output, as well as a $3.5 \mathrm{~mm}$ phono jack for audio. The Raspberry Pi 4 can run on various open source operating systems, including the official Raspbian.

\section{Arduino IDE}

The Arduino IDE is an open-source and userfriendly platform installed on a computer to communicate with Arduino-compatible microcontroller boards. It contains a whole range of features that simplifies the process of writing, uploading, compiling and debugging code for microcontroller boards [34]. The Arduino IDE is used in the proposed system to easily program the Arduino Pro Mini development board.

\section{thinger.io platform}

thinger.io [35] was selected as the Internet of Things (IoT) platform. It allows users to quickly connect and manage IoT products to the platform. These can be controlled with easy-to-use admin consoles, and Representational state transfer application program interface (REST API). Although the thinger.io platform is an open source IoT platform, it provides a ready to use, scalable cloud infrastructure for connecting things.

\section{Google Drive}

Google Drive is an online storage platform that offers a free plan of 15 GB to its users. Because of its large storage size, Google Drive platform was chosen to upload real-time pictures from the camera through the Raspberry Pi 4.

\subsection{Seat Occupancy System}

The seat occupancy sensors used are based on Force Sensitive Resistors (FSR). The resistance of these sensors vary when a force is applied to the sensing area. As the force on the sensor is increased, the resistance decreases and viceversa. In its simplest layout configuration, an FSR can be considered as a two-terminal device that can essentially be treated as a variable resistor whose value is controlled by applied force. For preliminary laboratory testing, the FSR Model 406 from Adafruit [36] was used. It provides a sensing 
area of $40 \mathrm{~mm} \times 40 \mathrm{~mm}$ and a thickness of $0.46 \mathrm{~mm}$. It is also light and unobtrusive so that it can be easily and inconspicuously fitted under the seat covers. Once the tests were conclusive, dedicated automotive seat occupancy sensors were procured for implementation in the bus. A snapshot of one force resistive sensor, Model MG120, on a bus seat is shown in Figure 3.

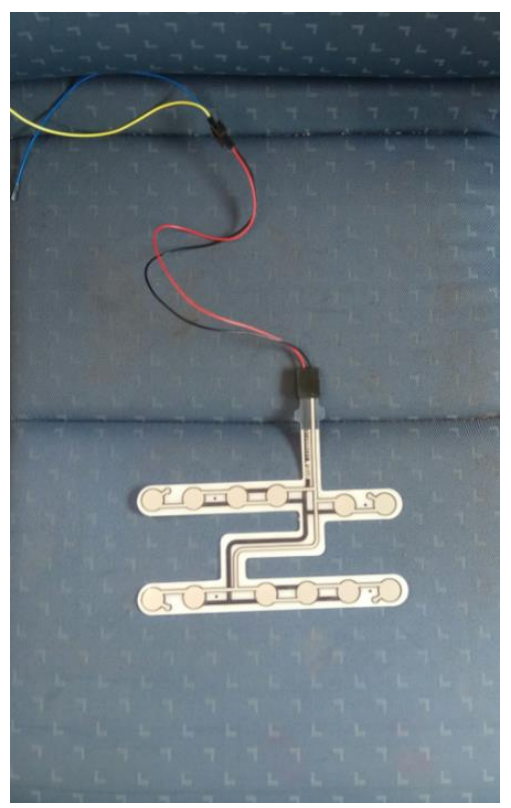

Fig. 3. Snapshot of FSR sensor on a bus seat

During the implementation, the FSR was inserted under the bus seat cover. Since the thickness of the FSR membrane is less than $1 \mathrm{~mm}$ and it is highly flexible, the latter was easily inserted under the seat cover. There is also a rear adhesive that keeps the membrane in place. As per the manufacturer's data sheet, the FSR can withstand 1 million activations and deactivations in its life time.

Although other sensors such as strain gauges, load cells and pressure transducers are more accurate than the FSR, the objective here is to detect the presence or absence of a weight, and not to actually measure the force applied. As such, the low-cost and ease of use of the FSR make it well suited for the proposed system.

Arduino Pro Mini microcontrollers [37] were used as interface between the Raspberry Pi and the FSR sensors, as shown in Figure 2. The Arduino Pro Mini is a low-cost open source microcontroller board developed by Arduino.cc and has an Atmega328 microcontroller incorporated on the board. It also accommodates 14 digital Input/Output (I/O) pins, out of which 6 pins are used for providing Pulse Width Modulation (PWM) outputs. Its major feature is its extremely compact size of $1.3 \times 0.7$ inch, enabling it to be housed in a small enclosure. This microcontroller is programmed through a Future Technology Devices International (FTDI) serial module.

All the Arduino microcontrollers as well as the Raspberry Pi were powered from a regulated 5V supply, derived from the vehicle's battery. The system architecture in Figure 2 shows that the Raspberry Pi acts as a Master whereas the FSRs are the Slaves. The Inter-Integrated Circuit (I2C) protocol is used for communication between the master and the slaves. Each I2C signal bus consists of two signals: Serial
Data Line (SDA) and Serial Clock Line (SCL). The Master node/device generates the clock for synchronization and initiates communication with slaves, while the slave node/devices receive the clock and respond when addressed by the master [38]. Theoretically, up to 127 slaves can work with one master, using 7-bit addressing mode. However in this scenario, we are using 20 slaves as the system was tested on a 20 -seater bus. Each slave is assigned a unique address by the master. The Arduino Pro Mini has an inbuilt Analog to Digital (A/D) converter, which is programmed to read the voltage values from resistances measured by the FSR. The connections from the FSR's to the Raspberry Pi were made using Ethernet Category 6 (Cat 6) cable, which is known to have a higher noise immunity, compared to previous versions of Ethernet cable. This cable is also widely available in hardware shops.

The following logic was implemented:

$$
\begin{array}{r}
\text { IF weight }<10 \mathrm{~kg}, \text { THEN output logic level ' } 0 \text { ' } \\
\text { ELSE output logic level ' } 1 \text { ' }
\end{array}
$$

Logic Level ' 0 ' implies seat is 'UNOCCUPIED', whereas Logic Level ' 1 ' implies that the seat is 'OCCUPIED'.

The $10 \mathrm{~kg}$ threshold has been selected to enable the system to discriminate between a passenger and a bag placed on a seat. The Raspberry Pi (Master) queries the slaves every 60 seconds, and the count is updated. Then, the number of occupied seats occupied is sent to the cloud platform, and updated every 60 seconds.

\subsection{Infrared (IR) Passenger Counting System}

A bi-directional passenger counting system was implemented using IR sensors connected to the Arduino Pro Mini microcontroller. The OPB100EZ low-cost sensor pair used in this system consists of an IR Light Emitting Diode (LED) and a phototransistor in separate casings. The sensor design is such that it does not require the light from the emitter and to the detector to go through a small aperture. Consequently, the sensor pair works accurately for separation distances of about $1 \mathrm{~m}$ even without precise alignment. Two pairs of IR sensors are used (IR1 and IR2), each pair consisting of an emitter (E) and a detector (D). Figure 4 indicates the arrangement of two sensor pairs placed near the entrance of a typical bus.

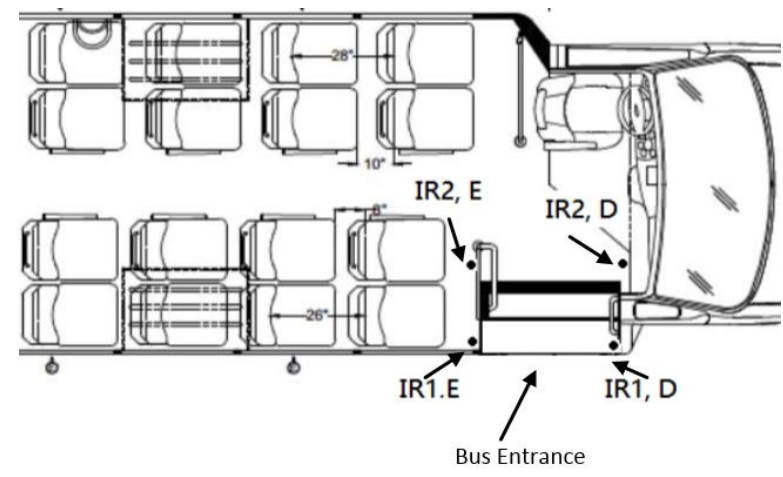

Fig. 4. Location of IR sensors at the bus entrance 
The flowchart given in Figure 5 details the sequence of events involved in determining the passenger count. Each emitter sends out an IR beam to its corresponding receiver. When a passenger passes between the sensor pair, the IR signal detected by the receiver is interrupted during a short interval. If the passenger is boarding the bus, the IR1 beam will be interrupted first, followed by that of IR2. In contrast, the beams would be disrupted in the reverse order in the case of an alighting passenger. The Arduino Pro Mini then updates the passenger count accordingly, and sends the data to the Raspberry Pi, which pushes same to the IoT platform.

\subsection{Bus Tracking System}

The bus tracking system was implemented through the low-power Neo 7M GPS module that outputs precise localization information. Longitude and latitude data relating to the vehicle location are provided continuously to the Raspberry $\mathrm{Pi}$ 4. The geolocation data is subsequently uploaded to the cloud. The GPS coordinates are mapped to OpenStreetMap on the thinger.io platform so as to display the location of the vehicle in real-time.

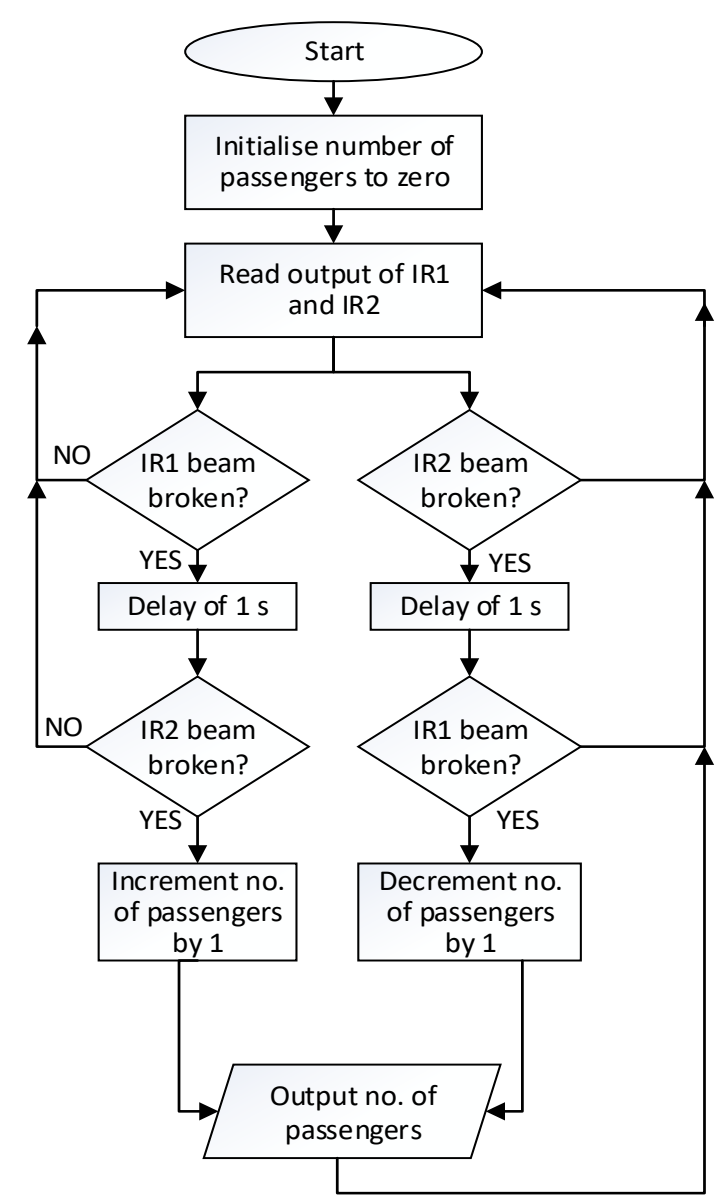

Fig. 5. Flowchart for the operation of IR Passenger Counting system

\subsection{Real Time Video Surveillance System}

The surveillance system uses a USB camera connected to the Raspberry Pi 4 board with the original Raspbian OS. Pictures are taken by the camera every two minutes using
Open Computer Vision Library (OpenCV) [39]. The latter is a library that is commonly used for processing and analysing video and images. Python scripts are written to take pictures and subsequently upload them to a Google Drive account. Given that Google Drive offers $15 \mathrm{~GB}$ of free storage, the frequency at which pictures were taken as well as their resolution were set so as to optimize the storage capacity. Thus, snapshots, in the Portable Network Graphics (png) [40] format were taken inside the bus every two minutes at a resolution of $800 \times 600$ pixels. These settings not only allowed a reasonably clear image resolution that could help in validating the seat occupancy in the bus but also enabled a large number of images to be stored. A timestamp is added to each picture to facilitate data analysis. The surveillance system can also be useful in enhancing the safety of the passengers and the driver. Thus, in case a safety issue is suspected in the bus minor modifications in the program settings can be made easily by the user at any time for the system to provide more frequent images and at higher resolutions. This will enable better monitoring of the situation in the bus.

\subsection{Mobile App}

A mobile app was developed to display the seat occupancy information, the real-time location of the bus on a map and the number of passengers in the bus as per the readings of the IR sensors. The app was built using the open source Apache Cordova framework [41]. Cordova is a framework that translates HTML/CSS/JS codes into Java which is used on the android platform. Cordova also enables the development of a hybrid app that could run on iOS and Android.

The mobile app is an essential component of a passenger information system (PIS). This typically ensures that bus passengers are kept informed about the route, connections, bus arrival time, and even travel disruption. Such information is very beneficial to passengers, since it allows better planning of trips, drastic reduction of waiting times on bus stops as well as increased passenger satisfaction. In the context of this work, the mobile app was designed to be used principally by bus operators rather than by passengers. Nevertheless, different views were designed for each category, based on login credentials. Herein, focus has been made on a mobile app that displays the bus identification (ID), the bus location and the number of occupied seats. These are readily retrieved from the thinger.io IoT platform and fed to the mobile app development platform. A snapshot of the mobile app homepage and options page is given in Figure 6. 


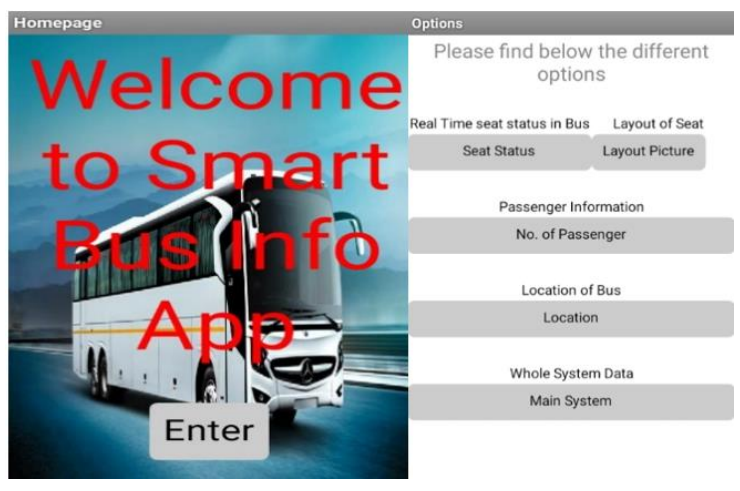

Fig. 6. Screenshot of app: Homepage (Left) and Options (Right)

\section{Testing and Results}

BusSIT was implemented and evaluated in a typical 20seater bus in Mauritius. Initially, each component of the system was tested separately. Once these tests were conclusive, integrated system testing was performed for one week, in different parts of Mauritius. Twelve trips were conducted during the test period. Figure 7 shows a snapshot illustrating the GPS coordinates during the various trips around the island from the thinger.io platform. It was found that the four sub-systems worked perfectly during all trips. In particular, the output from the IR passenger count sub-system concurred with seat occupancy sub-system at all instants during the twelve trips. Thus, $100 \%$ accuracy was obtained for the four sub-systems during the one-week testing period.

The following sections elaborate on the test results for each sub-system obtained for one testing instance. The latter refers to a trip from Reduit to Centre de Flacq, which usually takes about 50 minutes by bus.

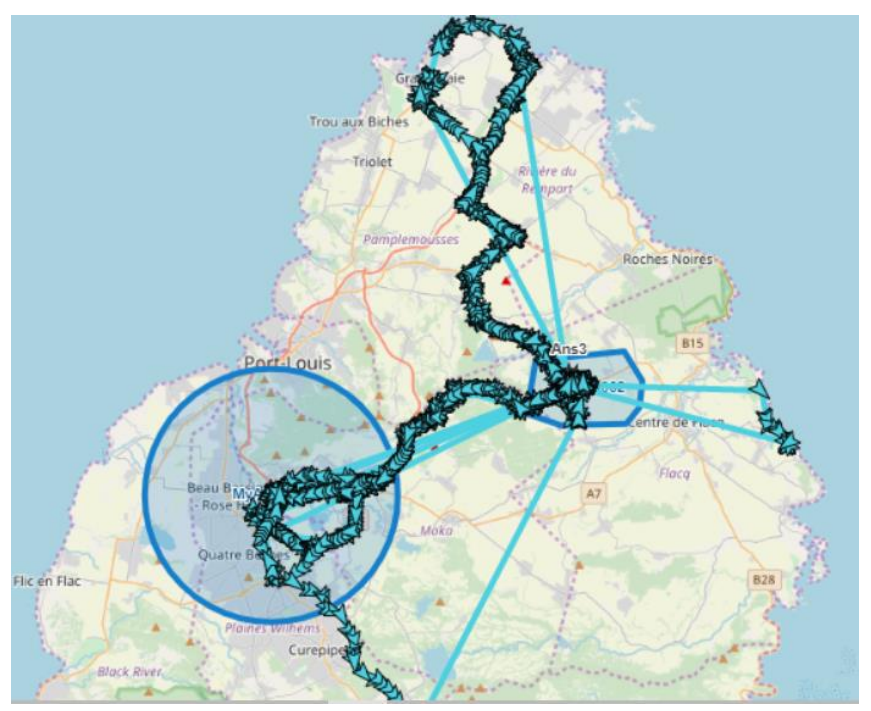

Fig 7. GPS coordinates from thinger.io platform for a period of one week

\subsection{Seat Occupancy System}

To validate the efficiency of the seat occupancy system, the number of passengers alighting and boarding the bus was manually logged at all times. The bus started from Reduit with no seated passengers, and at Central Flacq, all the passengers alighted the bus. Eight bus stops along the Reduit to Central Flacq route were taken into account during the tests. The online dashboard updates data received from seat sensors every 60 seconds. The output from the seat sensors during a typical test is shown on Figure 8. The $\mathrm{x}$-axis displays the time elapsed since the start of the trip while the tick marks indicate the times when the bus stopped at bus stops.

Although the seat occupancy system was tested independently, its output was validated with that of the IR Passenger Count system when only seated passengers were present in the bus. The seat occupancy displayed on the IoT platform matched the number of seated passengers at all times during the tests. This proved that the FSR sensors, and the whole setup were working reliably.

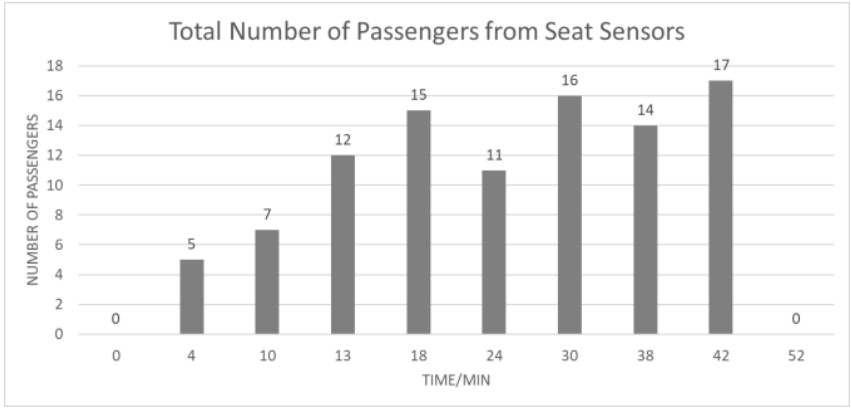

Fig. 8. Display of seat occupancy during a test trip

The main limitation of the system relates to its inability to precisely discriminate between a passenger and an object placed on the seat. The existing literature on this topic does not feature low-cost solutions. This issue was addressed to some extent by setting the weight detection threshold to 10 $\mathrm{kg}$, as detailed in Section 4.3. Bags rarely exceed this weight while young children usually weigh more than $10 \mathrm{~kg}$ and infants normally sit on their parent's lap.

\subsection{Infrared (IR) Passenger Counting System}

The passenger count was found to be accurate whenever passengers were moving into or out of the bus, lined up in an orderly manner. However, when two passengers were very close to each other, the count was updated by 1 only. To overcome this problem, the distance between the two sensor pairs was adjusted through trial and error. If they are too far apart, another passenger could step between the first sensor pair before the previous one has crossed the second sensor pair. Such a scenario would result in the system overlooking a passenger in the count. Moreover, a delay, computed on the basis of the $80 \mathrm{MHz}$ microcontroller frequency, was also included in the program to account for the minimum time that the passenger takes to move from one sensor pair to the other. This measure prevents the system from incrementing the count when luggage carried by a passenger crosses the sensor pairs. During the tests, it was found that a separation distance ranging from 35 to $42 \mathrm{~cm}$ between the sensor pairs resulted in accurate passenger count in almost all scenarios. The only time when the system failed to output a correct count occurred when a passenger crossed the sensor pairs while holding a child in his/her arms. Nevertheless, such children did not usually have a separate seat in the bus. It must also be noted 
that the passage near the bus door is narrow and does not allow two passengers to board or alight the bus side by side. The number of passengers entering and exiting the bus, measured by the IR sensors, is displayed in real-time on the Android mobile app. Results for the test trip described in Section 5.1 are shown in Figure 9. It can be seen that after driving for four minutes, 5 passengers boarded the bus at the first bus stop. After 10 minutes, the bus reached the second bus stop where 3 passenger alighted and 5 passengers boarded the bus. Hence, a passenger count of 7 was displayed on the IoT platform. The test trip lasted 52 minutes. It can be observed that the data on the resultant number of passengers, from the IR count sub-system corroborates with the output of the seat sensor sub-system shown in Figure 8. Additional key data that can be inferred from the display include arrival and departure times at bus-stops as well as the passenger flow. Passenger statistics in bus during each trip can be used to verify the revenues arising from ticket sales.

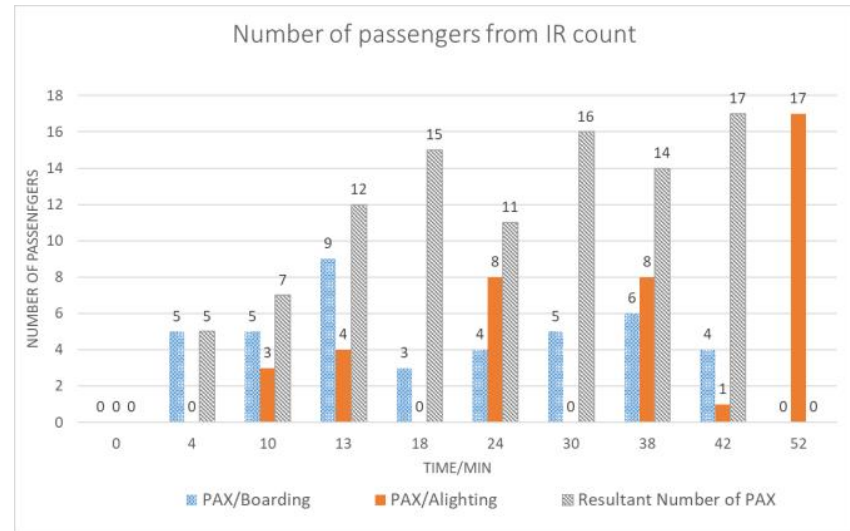

Fig. 9. Display of passengers boarding, alighting and sitting in the bus

\subsection{Bus Tracking System}

The bus tracking sub-system was tested independently in the bus during typical trips from Reduit to Centre de Flacq in Mauritius. Figure 10 shows the output of the bus tracking system on thinger.io during a test trip. The arrows show the itinerary followed by the bus from the test trip to its actual location in real-time. Meanwhile, the detailed logs of the geolocation data of the bus along with the corresponding timestamps are stored on the cloud. Important information can be inferred from a thorough analysis of the logs. These include identification of locations where stops were made and their duration as well as the average speed of the bus between consecutive stops.

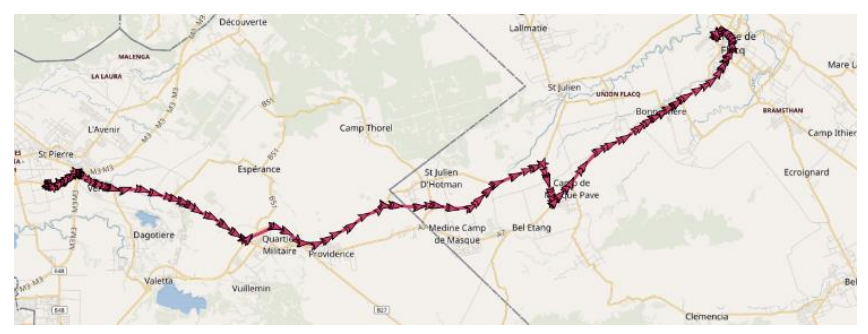

Fig. 10. GPS output displayed in thinger.io

\subsection{Video Surveillance System}

The camera was installed at the top of the bus front facing the back and adjusted so as to capture an overall view of all the seats. The video surveillance system uploaded pictures at intervals of two minutes. It is observed that the picture can be used to verify the output of the seat occupancy and passenger count systems, as the number of passengers can be counted manually from the picture. As mentioned in Section 4.5, if any problem is identified in the picture, the capture frequency can be increased so that the footage of the incident can either be viewed in nearly real-time or used by relevant authorities later.

\subsection{Comparison with existing systems}

The proposed BusSIT system was compared with commercially available seat management systems. BusSIT offers similar features to most existing systems. Thus, it detects seat occupancy and provides the data in near real-time to bus service providers and passenger information systems. Also, the proposed system is modular, fully scalable and requires low maintenance. On the other hand, most existing commercial systems interface their sensors with the onboard computer of the bus. This is a major limitation for buses which are not equipped with onboard computers, as is the case in many public vehicles in developing countries. BusSIT uses low-power embedded systems to integrate processing and wireless communication capabilities into the bus components.

In addition, BusSIT integrates additional features that support and supplement the seat occupancy system. They include camera surveillance, passenger counting and bus tracking. Commercial systems usually charge additional fees for implementing these features.

Another limitation of some existing systems is that they are applicable to typical buses of the country in which they are developed. However, buses differ considerably in their interior layout. For example, some buses allow standing passengers while others have separate doors for alighting and boarding passengers or have different seating arrangements. The proposed system has been designed so that it can be easily adapted to these different configurations. Since two different systems are used for passenger count and seat occupancy, the number of standing passengers can be inferred from the difference between the outputs of the passenger counting and seat occupancy systems. A necessary condition for correct operation is that passengers should not be allowed to stand near the IR sensor pairs. This should not be a problem as the IR sensors are installed near the door passage. For safety reasons, passengers are usually not allowed to stand in this area. BusSIT can also be easily adapted to buses with two doors. It only requires that the IR-based passenger count system to be duplicated along the passage of the other door. Furthermore, since each seat is fitted with a dedicated FSR sensor, the seat occupancy system can adapt to any seating configuration, with varying number of rows or columns. Each FSR sensor transmits the occupancy status of its seat to the Raspberry Pi through the Arduino Pro Mini.

As detailed in the cost breakdown provided in Table 1 and Table 2 respectively, the total capital cost of implementing 
BusSIT on the 20 -seater bus amounted to $\$ 505$ while the recurrent monthly costs amounted to $\$ 28$. The business plan quoted for the thinger.io IoT platform in Table 2 enables up to 100 devices, 100 dashboards, and 100 endpoints. At a monthly cost of $\$ 22.5$ only, the business plan largely exceeds the requirements of BusSIT. The data retention period is 3 years, and community/email support is provided [35]. The cost of the proposed system compares extremely favourably with commercial passenger counting system units which cost between $\$ 2,500$ and $\$ 10,000$ per bus [42]. It must be noted that the latter do not feature additional sub-systems included in BusSIT, such as real-time camera surveillance and bus tracking, as well as recurrent connectivity and maintenance costs.

Table 1 Cost breakdown for the capital cost of the BusSIT system.

\begin{tabular}{|l|c|c|c|}
\hline \multicolumn{1}{|c|}{ Item } & $\begin{array}{c}\text { Quantit } \\
\mathbf{y}\end{array}$ & $\begin{array}{c}\text { Unit } \\
\text { Cost/\$ }\end{array}$ & $\begin{array}{c}\text { Total } \\
\text { cost/USD }\end{array}$ \\
\hline $\begin{array}{l}\text { Raspberry Pi 4 } \\
\text { Model B (4GB } \\
\text { RAM) }\end{array}$ & 1 & 75 & 75 \\
\hline IR Sensors & 2 pairs & 6.5 & 13 \\
\hline USB Camera & 1 & 25 & 25 \\
\hline FSR sensor & 20 & 10 & 200 \\
\hline GPS Sensor & 1 & 15 & 15 \\
\hline Arduino Pro Mini & 22 & 2.6 & 57 \\
\hline 3G/4G USB dongle & 1 & 20 & 20 \\
\hline $\begin{array}{l}\text { Ethernet cables, } \\
\text { casings }\end{array}$ & lot & 100 & 100 \\
\hline Total & & & $\mathbf{5 0 5}$ \\
\hline
\end{tabular}

Table 2 Monthly (Recurrent) costs of BusSIT system

\begin{tabular}{|l|c|c|}
\hline Item & $\begin{array}{c}\text { Features - } \\
\text { Monthly Plan }\end{array}$ & Cost /USD \\
\hline $\begin{array}{l}\text { IoT Platform } \\
\text { (thinger.io) }\end{array}$ & BUSINESS Plan & 22.5 \\
\hline Internet subscription & $1 \mathrm{~GB}$ & 8 \\
\hline Total & & $\mathbf{2 8}$ \\
\hline
\end{tabular}

\section{Conclusion and Further Work}

The aim of this work was to design and implement a low-cost system that provides real-time passenger count data to bus operators. To this end, BusSIT was implemented, with supplementary features such as real-time GPS tracking, near real-time seat occupancy, and periodic images for verification. The system was built using open-source hardware, opensource software, and open IoT platforms, which contributed to its low cost. A mobile app was also developed to enable easy visualization of data from the online platforms. Tests in real-life conditions showed that the passenger counting system accurately displayed the number of commuters travelling in the bus during all trips. It is important to carefully adjust the distance between the two IR sensor pairs used in the system. This eliminates errors in the count arising when passengers are very close to each other while moving in or out of the bus. The seat occupancy system displayed the number of passengers correctly as well as the evolution of the passenger count during the trip. The output of the two systems could be viewed on the thinger.io platform in real-time. The passenger count can further be validated with the camera surveillance system which uploads images of the bus interior every two minutes. This system can also be used for passenger safety whereby its image frequency can be increased. Finally, the itinerary of the bus is tracked in realtime during trips by the GPS tracking system.

The system was tested successfully during a one-week period when twelve trips were carried out throughout Mauritius. Data collected by the various sub-systems include the number of passengers and seat usage in the bus at any time throughout the day, week or year. Bus location is also displayed and recorded for analysis on the thinger.io IoT platform. Also the camera installed with the Raspberry Pi upload photographs of the bus interior every two minutes. They can be used to provide a visual status report of each seat in the bus. All the data can be used to create maps displaying the location of each bus stop and historic data about the number of passengers boarding in and out at each bus stop. Seat occupancy patterns could also be analysed by bus stop, while the estimated arrival times of the bus to each bus stop could be estimated based on the current speed of the bus coupled with historic speed data series for that specific trip. Furthermore, since GPS data is also being recorded, more indepth studies could be carried out pertaining to journey time variation and schedule adherence.

The current BusSIT system has been developed as a proof-ofconcept prototype. Real world testing has been performed in a single 20 -seater bus. Once the system has evolved into a finished product, it could be deployed in bus fleets in order to achieve several benefits for passengers, bus companies and road network managers. First, it could be used to develop operational tools for bus operators. For example, additional buses could be inserted into the schedule at times when the system data reveal that buses are running late and/or are particularly crowded. Similarly, when buses are considerably delayed by traffic at particular locations, subsequent buses on the route could be advised to use alternative paths to bypass these locations. Thus, high quality live data has the potential to make scheduled buses more operationally resilient and more responsive to the needs of the passengers.

Second, passengers could additionally benefit from the enhanced operational data made available to them through internet-based passenger information portals - most likely through smartphone apps and large screens at heavily-used bus stops. Previous studies have shown that the provision of passenger information systems on smartphones apps has considerably enhanced the commuter journey experience due to the ubiquity of smartphones along with the real-time and interactive nature of the information presented [43], [44]. 
Third, bus operators will benefit from an improved control over the use of their resources. For example, tracking and identification of unofficial pickups/drops by bus drivers is difficult to detect for bus operators. Likewise, total income of the bus cannot be verified if tickets are not issued to passengers. The bus tracking module combined with the seat occupancy and passenger count systems in BusSIT enable bus operators to get the seat occupancy, passenger count, pickup/drops, stops and itinerary details both in real-time and historically.

Fourth, deployment of the proposed system in numerous buses will supply a wealth of passenger count and bus speed data along specific routes. This information could be used by the relevant authorities to corroborate traffic information and identify traffic hotspots on a specific route. Moreover, artificial intelligence algorithms could process historical passenger and traffic data gathered from all BusSIT systems to develop appropriate mitigation measures, such as to advise drivers to use alternative routes, suggest passengers to travel at different times, etc.

Finally, the aggregated data could also be used by bus operators for longer-term planning - to inform timetabling, driver and vehicle rostering schedules; route alterations; depot re-location plans; and vehicle design and investment strategies.

\section{References}

[1] O. Cats, J. West, and J. Eliasson, “A dynamic stochastic model for evaluating congestion and crowding effects in transit systems," Transp. Res. Part B Methodol., vol. 89, pp. 43-57, Jul. 2016.

[2] D. A. Hensher, W. H. Greene, and Z. Li, "Embedding risk attitude and decision weights in non-linear logit to accommodate time variability in the value of expected travel time savings," Transp. Res. Part B Methodol., vol. 45, no. 7, pp. 954-972, 2011.

[3] Z. Li and D. A. Hensher, "Crowding and public transport: A review of willingness to pay evidence and its relevance in project appraisal," Transp. Policy, vol. 18, no. 6, pp. 880-887, 2011.

[4] I. Essadeq, E. Dubail, and E. Jeanniere, "Modelling Passenger Congestion in Transit System Benchmark and Three Case Studies," Transp. Res. Procedia, vol. 14, pp. 1792-1801, 2016.

[5] D. K. Boyle, Passenger Counting Systems. Washington, D.C.: National Academies Press, 2009.

[6] X. J. Harmony and V. V Gayah, "Evaluation of Real-Time Transit Information Systems: An information demand and supply approach," Int. J. Transp. Sci. Technol., vol. 6, no. 1, pp. 86-98, 2017.

[7] U. Demir Alan and D. Birant, "Server-Based Intelligent Public Transportation System with NFC,"
IEEE Intell. Transp. Syst. Mag., vol. 10, no. 1, pp. 30-46, 2018.

[8] K. H. Kim and K. Lee, "Deep-Learning Seat Selection on a Tour Bus Based on Scenery and Sunlight Information," J. Comput. Sci. Eng., vol. 13, no. 3, pp. 89-98, 2019.

[9] K. Bauer, T. Bosker, K. N. Dirks, and P. Behrens, "The impact of seating location on black carbon exposure in public transit buses: Implications for vulnerable groups," Transp. Res. Part D Transp. Environ., vol. 62, pp. 577-583, 2018.

[10] M.-P. Pelletier, M. Trépanier, and C. Morency, "Smart card data use in public transit: A literature review," Transp. Res. Part C Emerg. Technol., vol. 19, no. 4, pp. 557-568, 2011.

[11] S. Wei et al., "Exploring the potential of open big data from ticketing websites to characterize travel patterns within the Chinese high-speed rail system," PLoS One, vol. 12, no. 6, pp. e0178023-e0178023, Jun. 2017.

[12] L.-M. Kieu, A. Bhaskar, and E. Chung, "A modified Density-Based Scanning Algorithm with Noise for spatial travel pattern analysis from Smart Card AFC data," Transp. Res. Part C Emerg. Technol., vol. 58, pp. 193-207, 2015.

[13] J. Zhao, Q. Qu, F. Zhang, C. Xu, and S. Liu, "Spatio-Temporal Analysis of Passenger Travel Patterns in Massive Smart Card Data," IEEE Trans. Intell. Transp. Syst., vol. 18, no. 11, pp. 3135-3146, 2017.

[14] M. Trépanier and C. Morency, Assessing Transit Loyalty with Smart Card Data. 2010.

[15] Y. Liu and T. Cheng, "Understanding public transit patterns with open geodemographics to facilitate public transport planning," Transp. A Transp. Sci., vol. 16, no. 1, pp. 76-103, Dec. 2020.

[16] M. R. Baltes and J. Rey, "The " Ins and Outs " of APCs : An Overview of Automatic Passenger Counters," J. Public Transp., vol. 2, no. 2, pp. 4764, 1999.

[17] A. Rakebrandt, "Transit Tracking: Automatic Passenger Counting Systems and Tracking Ridership," Mass Transit, vol. 33, no. 1, pp. 28-34, 2007.

[18] Thameslink, "Introducing the Siemens Class 700 fleet into service."

[19] K. Hashimoto, K. Morinaka, N. Yoshiike, C. Kawaguchi, and S. Matsueda, "People count system using multi-sensing application," in Proceedings of International Solid State Sensors and Actuators 
Conference (Transducers '97), 1997, vol. 2, pp. 1291-1294 vol.2.

[20] M. Trépanier and T. Yamamoto, "Workshop Synthesis: System Based Passive Data Streams Systems; Smart Cards, Phone Data, GPS," Transp. Res. Procedia, vol. 11, pp. 340-349, 2015.

[21] T. Oransirikul, R. Nishide, I. Piumarta, and H. Takada, "Measuring Bus Passenger Load by Monitoring Wi-Fi Transmissions from Mobile Devices," Procedia Technol., vol. 18, pp. 120-125, 2014.

[22] A. S. Zeeman, M. J. Booysen, G. Ruggeri, and B. Laganá, "Capacitive seat sensors for multiple occupancy detection using a low-cost setup," in 2013 IEEE International Conference on Industrial Technology (ICIT), 2013, pp. 1228-1233.

[23] N. H. H. Huy, N. Gulati, Y. Lee, and R. K. Balan, "Real-time detection of seat occupancy and hogging," in IoT-App 2015 - Proceedings of the 2015 International Workshop on Internet of Things Towards Applications, co-located with SenSys 2015, 2015, pp. 29-34.

[24] A. A. Atayero, O. A. Ilori, and M. O. Adedokun, "Development of seatsense: A wireless sensor network based seat detection system," Lect. Notes Eng. Comput. Sci., vol. 2220, pp. 711-714, 2015.

[25] H. Sterner, W. Aichholzer, and M. Haselberger, "Development of an Antenna Sensor for Occupant Detection in Passenger Transportation," Procedia Eng., vol. 47, pp. 178-183, 2012.

[26] C. Chen, Y. Chang, T. Chen, and D. Wang, "People Counting System for Getting In/Out of a Bus Based on Video Processing," in 2008 Eighth International Conference on Intelligent Systems Design and Applications, 2008, vol. 3, pp. 565-569.

[27] D. Liciotti, A. Cenci, E. Frontoni, A. Mancini, and P. Zingaretti, “An intelligent RGB-D video system for bus passenger counting," in Advances in Intelligent Systems and Computing, 2017, vol. 531, pp. 473-484.

[28] T. Yang, "Clustering method for counting passengers getting in a bus with single camera," Opt. Eng., vol. 49, no. 3, p. 037203, Mar. 2010.

[29] P. Verma and J. S. Bhatia, "Design and Development of GPS-GSM Based Tracking System with Google Map Based Monitoring," Int. J. Comput. Sci. Eng. Appl., vol. 3, pp. 33-40, Jun. 2013.

[30] W. Elmedany, A. Al-Omary, R. Al-Hakim, S. AlIrhayim, and M. Nusaif, A Cost Effective Real-Time Tracking System Prototype Using Integrated
GPS/GPRS Module. 2010.

[31] P. B. Fleischer, A. Y. Nelson, R. A. Sowah, and A. Bremang, "Design and development of GPS/GSM based vehicle tracking and alert system for commercial inter-city buses," in 2012 IEEE 4th International Conference on Adaptive Science \& Technology (ICAST), 2012, pp. 1-6.

[32] I. Lita, I. B. Cioc, and D. A. Visan, "A New Approach of Automobile Localization System Using GPS and GSM/GPRS Transmission," in 2006 29th International Spring Seminar on Electronics Technology, 2006, pp. 115-119.

[33] "Raspberry Pi 4 Tech Specs." [Online]. Available: https://www.raspberrypi.org/products/raspberry-pi4-model-b/specifications/.

[34] “Arduino Software.” [Online]. Available: https://www.arduino.cc/en/main/software.

[35] “Thinger IoT Platform.” [Online]. Available: https://thinger.io/.

[36] "Square Force-Sensitive Resistor (FSR) - Interlink 406." [Online]. Available:

https://www.adafruit.com/product/1075.

[37] “Arduino Pro Mini." [Online]. Available: https://www.arduino.cc/en/Guide/ArduinoProMini.

[38] N. X. P. Semiconductors, "UM10204 I2C-bus specification and user manual," Semiconductors, vol. 3, no. June, pp. 1-50, 2014.

[39] “OpenCV." [Online]. Available: https://opencv.org/.

[40] "Portable Network Graphics." [Online]. Available: http://www.libpng.org/pub/png/.

[41] “Apache CORDOVA.” [Online]. Available: https://cordova.apache.org/.

[42] “Automated Passenger Counting (APC) Systems: How Do They Work?" [Online]. Available: https://www.liveabout.com/automated-passengercounting-apc-2798822.

[43] S. Beul-Leusmann, C. Samsel, M. Wiederhold, K. H. Krempels, E. M. Jakobs, and M. Ziefle, "Usability evaluation of mobile passenger information systems," in Lecture Notes in Computer Science (including subseries Lecture Notes in Artificial Intelligence and Lecture Notes in Bioinformatics), 2014, vol. 8517 LNCS, no. PART 1, pp. 217-228.

[44] L. Kocher, "The mobile revolution in public transport," 2015. 